\title{
Factor V Leiden Mutation and Its Relation to Left Atrial Thrombus in Chronic Nonrheumatic Atrial Fibrillation
}

\author{
Mustafa GÖKÇE, ${ }^{1} \mathrm{MD}$, Fahri UÇAR, ${ }^{2} \mathrm{PhD}$, Mehmet KÜÇÜKOSMANOGLU, ${ }^{1} \mathrm{MD}$, \\ Turan ERDOĞAN, ${ }^{1} \mathrm{MD}$, and Şahin KAPLAN, ${ }^{1} \mathrm{MD}$
}

\begin{abstract}
SUMMARY
The genetic defect of coagulation factor $\mathrm{V}$, known as factor $\mathrm{V}$ Leiden, produces a resistance to degradation by activated protein $\mathrm{C}$ and increased venous thrombosis. However, the role of factor $\mathrm{V}$ Leiden in the formation of left atrial thrombus with nonrheumatic atrial fibrillation has not been studied. We investigated whether factor $\mathrm{V}$ Leiden is a risk factor for left atrial thrombus in patients with nonrheumatic atrial fibrillation. We analyzed clinical, echocardiographic, and biochemical data in 105 consecutive patients with nonrheumatic atrial fibrillation. These patients were divided into two groups; group A $(n=37)$ with left atrial thrombus and group $\mathrm{B}(n=68)$ without left atrial thrombus. The study also included 42 control subjects. Left atrial thrombus was investigated by using both transthoracic echocardiography and transesophageal echocardiography. Blood samples from the patients and controls were analyzed for the factor $\mathrm{V}$ Leiden mutation by DNA analysis, using the polymerase chain reaction. There was no significant difference in the prevalence of factor $\mathrm{V}$ Leiden between the patients and control subjects. The prevalence of factor V Leiden mutation was $8.1 \%$ (3/37) in patients with left atrial thrombus, and $8.8 \%(6 / 68)$ in patients without left atrial thrombus. The prevalence of factor $\mathrm{V}$ Leiden was $7.1 \%$ (3/42) in control subjects. The prevalance of factor V Leiden was $10 \%$ $(2 / 20)$ in patients with spontaneous echo contrast and $8 \%$ (7/85) in patients without spontaneous echo contrast. Multivariate analyses showed that left ventricular ejection fraction was an independent predictor of left atrial thrombus. Factor V Leiden mutation is not a risk factor for left atrial thrombus formation and spontaneous echo contrast in patients with nonrheumatic atrial fibrillation. (Jpn Heart J 2003; 44: 481-491)
\end{abstract}

Key words: Nonrheumatic atrial fibrillation, Left atrial thrombus, Spontaneous echo contrast

ATRIAL fibrillation results in stasis of blood flow in the left atrial appendage, leading to thrombus formation and embolic events such as stroke. ${ }^{1)}$ It is well known that the coagulative state in patients with chronic atrial fibrillation is higher than that in patients with normal sinus rhythm ${ }^{2-5)}$ and that the incidence of

From ${ }^{1}$ Department of Cardiology, ${ }^{2}$ Department of Genetics, KTÜ Faculty of Medicine.

Address for Correspondence: Mustafa GÖKÇE, MD, Department of Cardiology, KTÜ Faculty of Medicine, 61080 Trabzon/Turkey.

Received for publication September 2, 2002.

Revised and accepted November 7, 2002. 
thromboembolism is also significantly higher in these patients. ${ }^{6-8)}$ Transthoracic echocardiography is insensitive for the detection of left atrial thrombus, particularly for those smaller than $1 \mathrm{~cm}$ or confined to the left atrial appendage..$^{910}$ ) Transesophageal echocardiography (TEE) is known to be an excellent tool for the detection of lesions in and around the left atrial cavity. ${ }^{11-13)}$ Left atrial spontaneous echo contrast (LASEC) has become a common finding in patients with nonrheumatic atrial fibrillation since the clinical use of TEE. ${ }^{14-16)}$ This echo phenomenon is probably due to blood stasis and therefore is considered to presage thrombus formation. ${ }^{17)}$

Hemostatic factors could be important in the pathogenesis of atrial fibrillation-associated LASEC and thrombus formation. ${ }^{18-20)}$

The genetic defect of coagulation factor $\mathrm{V}$ known as factor $\mathrm{V}$ Leiden produces a resistance to degradation by activated protein C (APC) and increases the risk of venous thrombosis. ${ }^{21)}$ APC resistance is present in $20-60 \%$ of venous thrombosis cases. ${ }^{22)}$ Factor V Leiden is present in $4 \%$ to $6 \%$ of the general population. ${ }^{23)}$ It is caused by a single point mutation in the factor $\mathrm{V}$ gene in which arginine $(\mathrm{R})$ at position 506 is substituted with a glutamine (Q). ${ }^{24)}$ The relationship between factor V Leiden and thrombus formation in LA or LASEC with nonrheumatic atrial fibrillation has not been investigated until now.

The aim of this study was to investigate whether factor V Leiden is a risk factor for the formation of LA thrombus or LASEC in patients with nonrheumatic atrial fibrillation.

\section{MeThods}

Patient population: From July 1999 to March 2002, all patients diagnosed to have chronic nonrheumatic atrial fibrillation who were either admitted to the hospital or observed at our out-patient clinic were prospectively enrolled in this study. Inclusion criteria were atrial fibrillation rhythm which was persistent for $>30$ days as documented by serial electrocardiography, and the absence of physical or echocardiographic evidence of rheumatic mitral stenosis. Patients who had known esophageal disease or were unable to tolerate transesophageal study were excluded. In all, 105 consecutive patients were included and underwent transesophageal echocardiography.

They were 70 men and 35 women with a mean age of 63 years (range: 42$80)$. The underlying etiologies of atrial fibrillation were systemic hypertension $(n$ $=69)$, coronary artery disease $(n=30)$, idiopathic dilated cardiomyopathy $(n=4)$, chronic lung disease $(n=3)$, and lone atrial fibrillation $(n=3)$. At the time of transesophageal study, 11 patients were receiving anticoagulant therapy and 23 patients were receiving antiplatelet agents. A thorough history review was per- 
formed in all patients to search for thromboembolic events prior to transesophageal echocardiographic study. Twenty-two patients (21\%) were found to have a history of thromboembolism. Of these, 10 had transient ischemic attack, 9 ischemic stroke, and the remaining two had peripheral embolism. In all patients with ischemic stroke, the diagnosis of cerebral ischemia were further confirmed by computerized axial tomography. All peripheral embolic events were defined by angiography.

The patients were divided into two group according to whether or not they had thrombus in the LA. Patients with thrombus in the LA were defined as group A. This group consisted of 37 patients ( 24 men, 13 women, mean age $63 \pm 16$ ). Patients without thrombus in the LA were defined as group B. This group consisted of 68 patients (40 men, 28 women, mean age $61 \pm 19$ ).

Control group: Forty-two age and sex matched healthy subjects without heart disease formed the control group (28 men and 14 women; mean age, $62 \pm 15$, range, 41-72).

Echocardiography: All patients were studied by transthoracic and transesophageal echocardiography. Transthoracic studies were performed with a commercially available ultrasound system (Hewlett-Packard Sonus 5500) using a 2.5 or $3.5 \mathrm{mHz}$ phased array transducer. Multiple standard views were obtained in all patients and the control group. M-mode echocardiographic measurements were made according to the American Society of Echocardiography recommendation, ${ }^{25)}$ and left ventricular ejection fraction was calculated using the method of Teichholz, et al. $^{26)}$ Five consecutive beats were averaged for all M-mode measurements. Mitral regurgitation was assessed semiquantitatively by color flow imaging and was graded as mild, moderate, or severe. ${ }^{27)}$ Transesophageal echocardiography was performed using a 5-MHz multiplane imaging transducer (Hewlett Packard, model 21364A). All patients fasted for at least 6 hours. Intravenous sedation with diazepam $(2.5-5 \mathrm{mg})$ and topical pharyngeal anesthesia with $10 \%$ Xylocaine spray were administered before insertion of the transesophageal probe. The procedure was tolerated well by all patients and there were no complications. Left atrial thrombus was diagnosed by the presence of a well-circumscribed echo mass in the left atrial cavity or appendage. Particular care was taken to differentiate a thrombus from the pectinate muscles in the left atrial appendage. Left atrial spontaneous echo contrast was diagnosed by the presence of a dynamic cloud of amorphous echoes swirling slowly in the atrial cavity. When it was absent with usual gain and compression settings, the settings were systematically increased to increase the sensitivity of detection; however, efforts were taken to avoid noise artifact. Subsequently, the optimal gain and compression settings were obtained and used throughout the examination. The presence or absence of LASEC and thrombus was determined by two independent observ- 
ers who were unaware of the clinical history. When a discrepancy occurred, the video tape was reviewed until a consensus was reached.

Blood sampling and assay: Blood samples were drawn from cases and controls in the fasting state and collected in EDTA-tubes (Vacutaine, Becton Dickinson, Meylon, France) and then centrifuged at $2000 \mathrm{~g}$ for 20 minutes. DNA was extracted from blood using a nucleospin DNA extraction kit (Macherey-Nagel $\mathrm{GmbH}$, Düren, Germany). DNA concentrations were measured spectrophotometrically at $260 \mathrm{~nm}$ and stored at $-70^{\circ} \mathrm{C}$. All measurements were subsequently performed within 3 weeks of collection. Ten microliters of DNA was amplified by polymerase chain reaction (PCR) in a Techne-Genius thermocycler (TechneCambridge, UK) followed by digestion with the MnII restriction enzyme (New England Biolabs) at $37^{\circ} \mathrm{C}$ for 16 hours. The products were then subjected to metaphor agarose gel electrophoresis. After completion of electrophoresis, the gels were photographed under ultraviolet transillumination. The technicians were blinded to whether a specimen was from a case subject or a control subject.

Blood samples used to test the following haemostatic factors were obtained from patients at the time of admittance to the hospital before beginning the therapy. The samples were collected, centrifuged, and stored in appropriate tubes and under refrigerated conditions. Plasma concentrations of fibrinogen were measured by the immunonephelometric method using a commercial kit (Dade Behring, Liederbach, Germany). The D-dimer concentrations (Boehringer Mannheim, Germany) and von Willebrand factor (IMTEC immun-diagnostika $\mathrm{GmBH}$, Germany) were measured according to the manufacturer's instructions. Blood samples were also used for hemoglobin, hematocrit, platelet count, prothrombin time, and activated partial thromboplastin time measurements.

Statistical analysis: Results are expressed as the mean \pm SD. Continuous variables among groups were compared by one-way analysis of variance (ANOVA). Comparisons between the two groups were performed by means of an unpaired $t$ test for continuous variables. Categorical variables were analyzed with contingency tables using the chi square test and Fisher exact test when appropriate. Multivariate logistics regression analysis was performed to identify the clinical and echocardiographic risk factors for the presence of left atrial thrombus. For multiple regression, factors showing a value $P<0.1$ in univariate analysis were selected. A $P$ value $<0.05$ was considered statistically significant.

\section{RESULTS}

Patient and control characteristics: The baseline clinical variables of the groups are listed in Table I. Left atrium spontaneous echo contrast was present in 14 patients in group A and 6 patients in group B on TEE examination. 
Table I. Baseline Clinical and Echocardiographic Variables Among Patients Groups and Control Group

\begin{tabular}{lccc}
\hline & $\begin{array}{c}\text { Group A } \\
(n=37)\end{array}$ & $\begin{array}{c}\text { Group B } \\
(n=68)\end{array}$ & $\begin{array}{c}\text { Control Group } \\
(n=42)\end{array}$ \\
\hline Age (years) & $63 \pm 16$ & $61 \pm 19$ & $62 \pm 15$ \\
Men/Women & $20 / 17$ & $40 / 28$ & $28 / 14$ \\
Hypertension (\%) & $23(62 \%)$ & $42(61 \%)$ & $22(52 \%)$ \\
Diabetes mellitus (\%) & $11(29 \%)$ & $21(30 \%)$ & $10(24 \%)$ \\
Current tobacco smoking (\%) & $12(82 \%)$ & $20(29 \%)$ & $12(28 \%)$ \\
Embolic history (\%) & $9(24 \%)$ & $13(19 \%)$ & - \\
Coronary artery disease (\%) & $12(32 \%)$ & $18(26 \%)$ & $11(26 \%)$ \\
Duration of atrial fibrillation (\%) (years) & $6.1 \pm 5.8$ & $5.1 \pm 3.9$ & - \\
LAD (min) & $46 \pm 7$ & $43 \pm 4$ & $32 \pm 26 * *$ \\
LVEDD (mm) & $57 \pm 8$ & $53 \pm 7$ & $45 \pm 5^{* *}$ \\
LVEF (\%) & $46 \pm 12$ & $55 \pm 14 *$ & $59 \pm 18^{* * *}$ \\
$>$ M-MR (\%) & $3(8 \%)$ & $5(7 \%)$ & - \\
Spontaneous echo (\%) contrast & $14(37 \%)$ & $6(8 \%)^{*}$ & - \\
\hline
\end{tabular}

LAD = left atrial dimension; LVEDD = left ventricular end-diastolic dimension; $\mathrm{LVEF}=$ left ventricular ejection fraction; $>\mathrm{M}-\mathrm{MR}=$ moderate or severe mitral regurgitation, $* P<0.05$, between group $\mathrm{A}$ and $\mathrm{B}, * * P<0.05$, between control group and group $\mathrm{A}, \mathrm{B}, * * * P<0.05$, between control group and groups $\mathrm{A}$.

All thrombi in LA were detected by multiplane transesophageal echocardiography in all patients; however, only two thrombi which were located in the left atrial cavity were detected by transthoracic echocardiography in two patients.

Six thrombi (16\%) were located in the left atrial cavity, and $31(84 \%)$ were confined to the left atrial appendage.

All spontaneous echo contrasts were detected only by transesophageal echocardiography - transthoracic echocardiography did not detect any spontaneous echo contrasts. Of the 11 patients who were receiving anticoagulation therapy, left atrial thrombus was found in $2(18 \%)$ and spontaneous echo contrast was observed in $4(36 \%)$. The remaining patients who were receiving anticoagulant therapy had neither left atrial thrombus nor spontaneous echo contrast. Of the 23 patients who were receiving antiplatelet therapy, left atrial thrombus was observed in $6(26 \%)$ and spontaneous echo contrast was found in $10(43 \%)$. The percentage of patients with spontaneous echo contrast was higher in group A than group B [14 (37\%) in group A vs $6(8 \%)$ in group B, $P<0.05]$. While left atrial dimension and left ventricular end diastolic dimension were significantly higher in patients with atrial fibrillation than in the control group, left ventricular ejection fraction was significantly lower in group A than in the control group and group B. Except for spontaneous echo contrast, no other parameters were significantly different between groups $\mathrm{A}$ and $\mathrm{B}$. The percentage of patients with an 
embolic history was higher in group A than in group B, however, the difference was not statistically significant (24\% in group A vs $19 \%$ in group $\mathrm{B}, P>0.05)$.

Factor $V$ Leiden and haemostatic factors: Factor $V$ Leiden and haemostatic factors in group A, group B, and the control group are listed in Table II. A comparison of patients with and without thrombus in the LA and the control group revealed there were no significant differences in hematocrit, hemoglobin, prothrombin time, activated partial thromboplastin time, fibrinogen, D-dimer, white blood cell count, platelet count, or von Willebrand factor.

The prevalance of the factor $\mathrm{V}$ mutation was $8.1 \%(3 / 37)$ in patients with thrombus, $8.8 \%(6 / 68)$ in patients without thrombus, and $7.1 \%(3 / 42)$ in control subjects. There were no significant differences in factor V Leiden between the patients with atrial fibrillation who did or did not have thrombus and the control subjects.

Factor V Leiden, haemostatic factors and spontaneous echo contrast: The haemostatic factors and factor V Leiden in patients with spontaneous echo contrast and without spontaneous echo contrast are listed in Table III. In the comparison of patients with spontaneous echo contrast and those without spontaneous echo contrast, there were no differences in hematocrit, hemoglobin, prothrombin time, activated partial thromboplastin time, fibrinogen, D-dimer, white blood cell count, platelet count, or factor V Leiden. However, there was a statistically significant difference in von Willebrand factor between the two groups. Patients with spontaneous echo contrast had a higher percentage of von Willebrand factor than patients without spontaneous echo contrast (119.2 \pm 12.6 vs $95.6 \pm 13.2, P<$ $0.05)$.

Table II. Factor V Leiden and Haemostatic Factors

\begin{tabular}{lccc}
\hline & $\begin{array}{c}\text { Group A } \\
(n=37)\end{array}$ & $\begin{array}{c}\text { Group B } \\
(n=68)\end{array}$ & $\begin{array}{c}\text { Control Group B } \\
(n=42)\end{array}$ \\
\hline Hct $(\%)$ & $46.1 \pm 3.9$ & $45.7 \pm 4.1$ & $45.8 \pm 3.8$ \\
$\mathrm{Hb}(\mathrm{g} / \mathrm{dL})$ & $15.4 \pm 1.44$ & $15.1 \pm 1.38$ & $15.2 \pm 1.4$ \\
$\mathrm{PT}(\mathrm{s})$ & $12.0 \pm 0.69$ & $11.8 \pm 0.81$ & $12.2 \pm 0.74$ \\
aPTT $(\mathrm{s})$ & $30.1 \pm 2.11$ & $31.2 \pm 2.53$ & $30.4 \pm 2.24$ \\
Fibrinogen $(\mathrm{mg} / \mathrm{dL})$ & $241.3 \pm 43.5$ & $252.1 \pm 51.6$ & $246.8 \pm 49.8$ \\
D-dimer $(\mathrm{ng} / \mathrm{mL})$ & $141.3 \pm 38.3$ & $136.7 \pm 36.8$ & $133.5 \pm 41.6$ \\
White blood cells $\left(10^{3} / \mu \mathrm{L}\right)$ & $10.3 \pm 1.8$ & $10.8 \pm 1.6$ & $10.5 \pm 1.5$ \\
Platelet count $\left(10^{3} / \mu \mathrm{L}\right)$ & $301.5 \pm 44.1$ & $304.8 \pm 44.6$ & $308.7 \pm 49.6$ \\
Factor V Leiden $(\%)$ & $3(8.1 \%)$ & $6(8.8 \%)$ & $3(7.1 \%)$ \\
von Willebrand factor $(\%)$ & $108.6 \pm 3$ & $106.2 \pm 20.1$ & $103.4 \pm 19.8$ \\
\hline
\end{tabular}

$\mathrm{Hb}=$ hemoglobin Hct = hematocrit $\mathrm{PT}=$ prothrombin time; $\mathrm{aPTT}=$ activated partial thromboplastin time. 
Table III. Factor V Leiden and Haemostatic Factors in Patients With Spontaneous Echo Contrast and Those Without Spontaneous Echo Contrast.

\begin{tabular}{lccc}
\hline & $\begin{array}{c}\text { Spontaneous } \\
\text { Echo contrast }(+) \\
(n=20)\end{array}$ & $\begin{array}{c}\text { Spontaneous } \\
\text { Echo contrast }(-) \\
(n=85)\end{array}$ & $P$ \\
\hline Hct $(\%)$ & $47.2 \pm 2.8$ & $45.4 \pm 3.8$ & $\mathrm{NS}$ \\
$\mathrm{Hb}(\mathrm{g} / \mathrm{dL})$ & $15.6 \pm 1.2$ & $14.9 \pm 1.1$ & $\mathrm{NS}$ \\
PT $(\mathrm{s})$ & $11.9 \pm 0.80$ & $11.8 \pm 0.71$ & $\mathrm{NS}$ \\
aPTT (s) & $30.4 \pm 2.2$ & $30.8 \pm 2.4$ & $\mathrm{NS}$ \\
Fibrinogen $(\mathrm{mg} / \mathrm{dL})$ & $264 \pm 42.2$ & $258 \pm 47.4$ & $\mathrm{NS}$ \\
D-dimer $(\mathrm{ng} / \mathrm{mL})$ & $139.8 \pm 40.4$ & $135.6 \pm 34.7$ & $\mathrm{NS}$ \\
White blood cells $\left(10^{3} / \mu \mathrm{L}\right)$ & $105.2 \pm 1.9$ & $104.3 \pm 1.7$ & $\mathrm{NS}$ \\
Platelet count $\left(10^{3} / \mu \mathrm{L}\right)$ & $308.6 \pm 53.4$ & $302.8 \pm 45.6$ & $\mathrm{NS}$ \\
Factor V Leiden $(\%)$ & $2(10 \%)$ & $7(8 \%)$ & $\mathrm{NS}$ \\
von Willebrand factor $(\%)$ & $119.2 \pm 12.6$ & $95.6 \pm 13.2$ & $P<0.05$ \\
\hline
\end{tabular}

$\mathrm{Hb}=$ Hemoglobin; Hct = Hematocrit PT = Prothrombin time; $\mathrm{APTT}=$ Activated partial thromboplastin time; NS = Not significant.

\section{DISCUSSION}

Patients with nonrheumatic atrial fibrillation have approximately five times the risk of systemic embolization compared with those without this arrhythmia. ${ }^{8,28)}$ The increased thromboembolic incidence is due to thrombus formation in the left atrium.,29) Thrombus formation is preferentially found in the atrial appendage and has been thought to be induced by blood stasis associated with atrial fibrillation. ${ }^{30,31)}$ Various factors determine the individual risk for the development of left atrial clot and thromboembolization in patients with nonrheumatic atrial fibrillation. These factors include reduced peak left atrial appendage velocity, increased plasma markers of platelet activation-beta thromboglobulin, platelet factor 4, thrombin-antithrombin complexes, D-dimers, and von Willebrand factor. ${ }^{4,5,20,32)}$ However, factor $\mathrm{V}$ Leiden has not been investigated until now to determine whether it is a factor which contributes to the formation of left atrial thrombus. To our knowledge, this study is the first report to investigate the relation ship between factor V Leiden and left atrial thrombus in patients with nonrheumatic atrial fibrillation. Transthoracic echocardiography was unable to show all thrombi in the left atrium in all patients with atrial fibrillation. ${ }^{33)}$ In our study, transthoracic echocardiography revealed only two thrombi $(1.5 \%)$ in the left atrium in the patient groups $(n=105)$. In the present study using transesophageal echocardiography, we found a $35 \%$ prevalence of left atrial thrombus in patients with nonrheumatic atrial fibrillation. We also confirmed the superiority of transesophageal echocardiography for the detection of atrial thrombus. Eighty-four percent of the detected thrombi were confined to the left atrial appendage, which in most cases was easily assessed by transesophageal echocardiography but not by 
a transthoracic approach. The prevalence of transesophageal echo detected left atrial thrombus in our study is higher than that of previously studies. ${ }^{34,35)}$ The difference of the reported prevalence is probably due to different patient selection, and characteristics such as the percent age of patients who were receiving anticoagulation medication.

Factor V Leiden has recently emerged as an important cause of venous thrombosis, and it is associated with a single point mutation in the factor $\mathrm{V}$ gene (FVQ 506 or factor V Leiden), which removes an important cleavage site for activated protein $\mathrm{C}$. It is still unclear whether factor $\mathrm{V}$ Leiden predisposes patients to arterial thrombosis or left atrial thrombosis. One of the largest studies to investigate the relationship between atrial fibrillation and factor V Leiden is the SPAF III Study (1). In this study, the presence of the factor V Leiden mutation was not associated with thromboembolism. But this study only comprise low-risk atrial fibrillation patients who had low-cardioembolic risks, such as lower rate diabetes mellitus, hypertension, heart failure and all patients were given aspirin. However, our study group consisted of relatively high-risk atrial fibrillation patients who had high rates of hypertension, diabetes mellitus, and echocardiographic measurements (low LVEF, high LVEDD and LAD).

Hisar, $e a^{36}$ investigated the role of activated protein $\mathrm{C}$ resistance in left atrial thrombogenesis in patients with mitral stenosis. They found the presence of factor V Leiden is not an independent risk factor for left atrial thrombus formation in mitral stenosis, but it may increase the risk of left atrial thrombus formation when present in combination with atrial fibrillation. In their study, the study group was limited to patients with mitral stenosis. However, our study was conducted in patients with nonrheumatic atrial fibrillation.

The prevalence of factor $\mathrm{V}$ Leiden among 752 low-risk AF patients was reported to be $6 \%(n=46) .{ }^{1)}$ Hisar, et $a l^{36)}$ found that the prevalence of factor $\mathrm{V}$ Leiden was common in both patients with left atrial thrombus (23\%) and those without left atrial thrombus (16\%). In the present study, we found that the prevalence of factor V Leiden in patients with left atrial thrombus (8.1\%) and those without left atrial thrombus (8.8\%) was lower than in the Hisar study. This difference may be due to differences in patient selection, baseline characteristics, and underlying anatomical lesions. The main finding that emerged from this study is that factor $\mathrm{V}$ Leiden mutation is not a risk factor for the development of atrial thrombus in patients with atrial fibrillation since there was no difference between patients with left atrial thrombus and those without left atrial thrombus.

The presence of left atrial spontaneous echo contrast has been associated with an increased thromboembolic risk in patients with nonrheumatic atrial fibrillation. ${ }^{20,22)}$ Although several studies have described the presence of left atrial thrombus in patients with nonrheumatic atrial fibrillation, ${ }^{14,15,35)}$ the prevalence 
and clinical significance of left atrial thrombus in this disease entity are poorly understood. In our study, left atrial spontaneous echo contrast was detected in 20 of the 105 patients $(19 \%)$ by transesophageal echocardiography, but was not found in any patient by transthoracic echocardiography. Patients with spontaneous echo contrast had higher von Willebrand factor levels than those without spontaneous echo contrast $(119.2 \pm 12.6$ vs $95.6 \pm 13.2, P<0.05)$. This finding is similar to a previous study. ${ }^{20)}$

In the comparison of patients with and without left atrial spontaneous echo contrast, there were no significant differences in the prevalence of factor $\mathrm{V}$ Leiden [2 (10\%) patients with spontaneous echo contrast vs 7 (8\%) patients without spontaneous echo contrast, $P<0.05]$. There were no differences between the two groups with respect to the other haemostatic factors.

Multivariate analysis revealed that a left ventricular ejection fraction $<50 \%$ was an independent predictor for the presence of left atrial thrombus $(P=0.042$, odds ratio $=3.18,95 \% \mathrm{CI}=1.03-4.27$ ). But a factor $\mathrm{V}$ Leiden mutation was not an independent predictor for the presence of either left atrial thrombus or spontaneous echo contrast.

According to these findings, a factor $\mathrm{V}$ Leiden mutation is not a risk factor for left atrial thrombus formation in patients with nonrheumatic atrial fibrillation. Study limitations: The most important limitation of our study is the small number of patients studied. Large prospective studies are needed to establish whether the factor $\mathrm{V}$ Leiden mutation is a risk factor for left atrial thrombus formation in patients with nonrheumatic atrial fibrillation. The other limitation is patient selection for determining the presence of left atrial thrombus. It has been reported that left atrial thrombus can be resolved by warfarin therapy. ${ }^{37)}$ In our study, a significant number of patients $(10 \%)$ were on anticoagulation therapy at the time of the study, and this may have mistakenly lowered the prevalence of left atrial thrombus.

\section{ACKNOWLEDGMENT}

We are very grateful to Orhan Topsakal for his technical assistance.

\section{REFERENCES}

1. Feinberg WM, Pearce LA, Hart RG, et al. Markers of thrombin and platelet activity in patients with atrial fibrillation. Correlation with stroke among 1532 participants in the stroke prevention in atrial fibrillation III study. Stroke 1999; 30: 2547-53.

2. Kumagai K, Fukunami M, Ohmori M, Kitabatake A, Kamada T, Hori N. Increased intra-cardiovascular clotting in patients with chronic atrial fibrillation. JACC 1990; 16: 377-80. 
3. Uno M, Tsuji H, Sawada S, Toyoda T, Nakagawa M. Fibrino-peptide A (Fpa) level in atrial fibrillation and effect of heparin administration. Jpn Circ J 1988; 52: 9-12.

4. Yamaguchi K, Furui H, Taniguchi N, Sotobata I. Plasma $\beta$-thromboglobulin and platelet factor 4 concentrations in patients with atrial fibrillation. Jpn Heart J 1986; 27: 481-7.

5. Asakura H, Hifumi S, Jokaji H, et al. Prothrombin fragment F1+2 and thrombin-antithrombin III complex are useful remarkers of the hypercoagulable state in atrial fibrillation. Blood Coagul Fibrinolysis 1992; 3: 469-73.

6. Wolf PA, Dawber TR, Emerson T Jr, Kannel WB. Epidemiologic assessment of chronic atrial fibrillation and risk of stroke: the Framingham Study. Neurology 1978; 28: 973-7.

7. Sherman DG, Goldman L, Whiting RR, Jurgessen K, Kaste M, Easton JD. Thromboembolism in patients with atrial fibrillation. Arch Neurol 1984; 41: 708-10.

8. Hinton RC, Kistler JP, Fallon JT. Influence of etiology of atrial fibrillation on incidence of systemic embolism. Am J Cardiol 1977; 40: 509-13.

9. Shrestha NK, Moreno FL, Narciso FV, Torres L, Calleja HB. Two-dimensional echocardiographic diagnosis of left atrial thrombus in rheumatic heart disease: a clinicopathologic study. Circulation 1983; 67: 341-7.

10. Chiang CW, Pang SC, Lin FC, et al. Diagnostic accuracy of two-dimensional echocardiography for detection of left atrial thrombus in patients with mitral stenosis. J Ultrasound Med 1987; 6: 525-9.

11. Aschenberg W, Schlüter M, Kremer P, Schröder E, Siglow V, Bleifeld W. Transesophageal two-dimensional echocardiography for the detection of left atrial appendage thrombus. J Am Coll Cardiol 1986; 7: 163-6.

12. Hausmann D, Mügge A, Becht I, Daniel WG. Diagnosis of patent foramen ovale by transesophageal echocardiography and association with cerebral and peripheral embolic events. Am J Cardiol 1992; 70: 668-72.

13. Castello R, Pearson AC, Labovitz AJ. Prevalence and clinical implications of atrial spontaneous echo contrast in patients undergoing transesophageal echocardiography. Am J Cardiol 1990; 65: 1149-53.

14. Black IW, Hopkins AP, Lee LCL, Walsh WF. Left atrial spontaneous echo contrast: a clinical and echocardiographic analysis. J Am Coll Cardiol 1991; 18: 398-404.

15. Tsai LM, Chen JII, Lin LJ, Kwan CM. Clinical implications of left atrial spontaneous echo contrast in nonrheumatic atrial fibrillation. Am J Cardiol 1992; 70: 327-31.

16. Leung DYC, Black IW, Cranney GB, Hopkins AP, Walsh WF. Prognostic implications of left atrial spontaneous echo contrast in nonvalvular atrial fibrillation. J Am Coll Cardiol 1994; 24: 755-62.

17. Sigel B, Coelho JCU, Spigos DG, et al. Ultrasonography of blood during stasis and coagulation. Invest Radiol 1981; 16: 71-6.

18. Fukuchi M, Watanabe J, Kumagai K, et al. Increased von Willebrand factor in the endocardium as a local predisposing factor for thrombogenesis in overloaded human atrial appendage. JACC Vol. 37, No. 5, 2001; 143642.

19. Weiss HJ, Turitto VT, Baumgartner HR. Effect of shear rate on platelet interaction with subendothelium in citrated and native blood. I. Shear rate-dependent decrease of adhesion in von Willebrand's disease and the Bernard-Soulier syndrome. J Lab Clin Med 1978; 92: 750-64.

20. Sakariassen KS, Bolhuis PA, Sixma JJ. Human blood platelet adhesion to artery subendothelium is mediated by factor VIII-Von Willebrand factor bound to the subendothelium. Nature 1979; 279: 636-8.

21. Gowda MS, Zucker ML, Vacek JL, et al. Incidence of Factor V Leiden in patients with acute myocardial infarction. J Thromb Thrombolysis 2000; 9: 43-5.

22. Dahlback B. Resistance to activated protein C caused by the factor VR506Q mutation is a common risk factor for venous thrombosis. Thromb Haemost 1997; 78: 483-8.

23. Res DC, Cox MC, Clegg JB. World distribution of factor V Leiden. Lancet 1995; 346: 1133-4.

24. Bertina RM, Koeleman BP, Koster T, et al. Mutation in blood coagulation factor V associated with resistance to activated protein C. Nature 1994; 369: 64-7.

25. Sahn DJ, DeMaria A, Kisslo J, Weyman A. Recommendations regarding quantitation in M-mode echocardiography: results of a survey of echocardiographic measurements. Circulation 1978; 58: 1072-83.

26. Teichholz LE, Kreulen T, Herman MV, Gorlin R. Problems in echocardiographic volume determinations: echocardiographic-angiographic correlations in the presence or absence of asynergy. Am J Cardiol 1976; 37: 711.

27. Helmcke F, Nanda NC, Hsiung MC, et al. Color Doppler assessment of mitral regurgitation with orthogonal planes. Circulation 1987; 75: 175-83. 
28. Stroke Prevention in Atrial Fibrillation Committee on Echocardiography. Transesophageal echocardiographic correlates of thromboembolism in high-risk patients with atrial fibrillation. Ann Intern Med 1998; 128: 639-47.

29. Halperin JL, Hart RG. Atrial fibrillation and stroke: new ideas, persisting dilemmas. Stroke 1988; 19: 937-41.

30. Pollick C, Taylor D. Assessment of left atrial appendage function by transesophageal echocardiography. Implications for the development of thrombus. Circulation 1991; 84: 223-31.

31. Mügge A, Kühn H, Nikutta P, et al. Assessment of left atrial appendage function by biplane transesophageal echocardiography in patients with nonrheumatic atrial fibrillation: identification of a subgroup of patients at increased embolic risk. J Am Coll Cardiol 1994; 23: 599-607.

32. Heppell RM, Berkin KE, McLenachan JM, Davies JA. Haemostatic and haemodynamic abnormalities associated with left atrial thrombosis in non-rheumatic atrial fibrillation. Heart 1997 May; 77: 407-11.

33. Lovett JL, Sandok BA, Giuliani ER, Nasser FN. Two-dimensional echocardiography in patients with focal cerebral ischemia. Ann Intern Med 1981; 95: 1-4.

34. Tsai LM, Lin LJ, Teng JK, Chen JH. Prevalence and clinical significance of left atrial thrombus in nonrheumatic atrial fibrillation. International Journal of Cardiology 1997; 58: 163-9.

35. Brown J, Sadler DB. Left atrial thrombus in non-rheumatic atrial fibrillation: assessment of prevalence by transesophageal echocardiography. Int J Cardiac Imaging 1993; 9: 65-72.

36. Hisar I, Ileri M, Yetkin E, et al. Role of activated protein C resistance in left atrial thrombogenesis in patients with mitral stenosis. Angiology 2000; 51855-60.

37. Tsai LM, Hung JS, Chen JH, Lin LJ, Fu M. Resolution of left atrial appendage thrombus in mitral stenosis after warfarin therapy. Am Heart J 1991; 121: 1232-4. 